\title{
Numerical Solutions of Fredholm Integral Equations Using Bernstein Polynomials
}

\author{
A. Shirin ${ }^{1}$ and M. S. Islam ${ }^{2}$ \\ ${ }^{1}$ Institute of Natural Sciences, United International University, Dhaka-1209, Bangladesh \\ ${ }^{2}$ Department of Mathematics, University of Dhaka, Dhaka-1000, Bangladesh
}

Received 23 February 2010, accepted in revised form 23 April 2010

\begin{abstract}
In this paper, Bernstein piecewise polynomials are used to solve the integral equations numerically. A matrix formulation is given for a non-singular linear Fredholm Integral Equation by the technique of Galerkin method. In the Galerkin method, the Bernstein polynomials are used as the approximation of basis functions. Examples are considered to verify the effectiveness of the proposed derivations, and the numerical solutions guarantee the desired accuracy.
\end{abstract}

Keywords: Fredholm integral equation; Galerkin method; Bernstein polynomials.

(C) 2010 JSR Publications. ISSN: 2070-0237 (Print); 2070-0245 (Online). All rights reserved.

DOI: $10.3329 /$ jsr.v2i2.4483

J. Sci. Res. 2 (2), 264-272 (2010)

\section{Introduction}

In the survey of solutions of integral equations, a large number of analytical but a few approximate methods for solving numerically various classes of integral equations [1, 2] are available. Since the piecewise polynomials are differentiable and integrable, the Bernstein polynomials $[4,5]$ are defined on an interval to form a complete basis over the finite interval. Moreover, these polynomials are positive and their sum is unity. For these advantages, Bernstein polynomials have been used to solve second order linear and nonlinear differential equations, which are available in the literature, e.g. Bhatti and Bracken [7]. Very recently, Mandal and Bhattacharya [6] have attempted to solve integral equations numerically using Bernstein polynomials, but they obtained the results in terms

\footnotetext{
${ }^{2}$ Corresponding author:mdshafiqul@yahoo.com
} 
of finite series solutions. In contrast to this, we solve the linear Fredholm integral equation by exploiting very well known Galerkin method [3] and Bernstein polynomials are used as trial functions in the basis. For this, we give a short introduction of Bernstein polynomials first. Then we derive a matrix formulation by the technique of Galerkin method. To verify our formulation we consider three examples, in which we obtain exact solutions for two examples even using a few and lower order polynomials. On the other hand, the last example shows an excellent agreement of accuracy compared to exact solution and convergence as well. All the computations are performed using MATHEMATICA.

\section{Bernstein Polynomials}

The general form of the Bernstein polynomials [4-7] of $n$th degree over the interval $[a, b]$ is defined by

$$
B_{i, n}(x)=\left(\begin{array}{l}
n \\
i
\end{array}\right) \frac{(x-a)^{i}(b-x)^{n-i}}{(b-a)^{n}}, a \leq x \leq b, \quad i=0,1.2, \cdots, n
$$

Note that each of these $n+1$ polynomials having degree $n$ satisfies the following properties:
i) $B_{i, n}(x)=0$, if $i<0$ or $i>n$,
ii) $\sum_{i=0}^{n} B_{i, n}(x)=1$;
iii) $B_{i, n}(a)=B_{i, n}(b)=0, \quad 1 \leq i \leq n-1$

Using MATHEMATICA code, the first 11 Bernstein polynomials of degree ten over the interval $[a, b]$, are given below:

$$
\begin{array}{ll}
B_{0,10}(x)=(b-x)^{10} /(b-a)^{10} & \left.B_{6,10}(x)=21 \varphi b-x\right)^{4}(x-a)^{6} /(b-a)^{10} \\
\left.B_{1,10}(x)=1 \varphi b-x\right)^{9}(x-a) /(b-a)^{10} & \left.B_{7,10}(x)=12 \emptyset b-x\right)^{3}(x-a)^{7} /(b-a)^{10} \\
B_{2,10}(x)=45(b-x)^{8}(x-a)^{2} /(b-a)^{10} & B_{8,10}(x)=45(b-x)^{2}(x-a)^{8} /(b-a)^{10} \\
B_{3,10}(x)=12(b-x)^{7}(x-a)^{3} /(b-a)^{10} & \left.B_{8,10}(x)=10 b-x\right)(x-a)^{9} /(b-a)^{10} \\
\left.B_{4,10}(x)=21 \varphi b-x\right)^{6}(x-a)^{4} /(b-a)^{10} & B_{0,10}(x)=(x-a)^{10} /(b-a)^{10} \\
\left.B_{5,10}(x)=25 x b-x\right)^{5}(x-a)^{5} /(b-a)^{10} &
\end{array}
$$

Now the first six polynomials over $[0,1]$ are shown in Fig. 1(a), and the remaining five polynomials are shown in Fig. 1(b). 


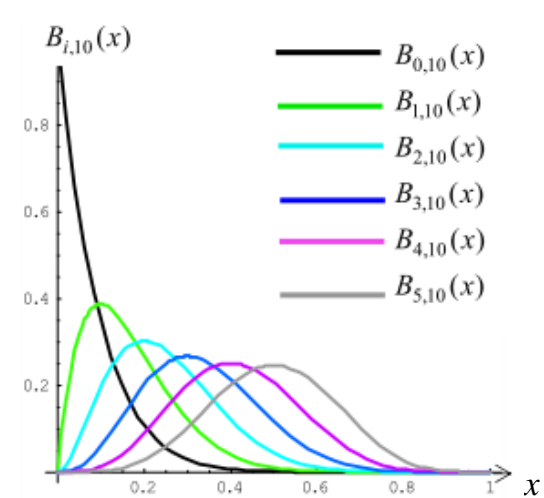

Fig. 1(a). Graph of first 6 Bernstein polynomials over $[0,1]$.

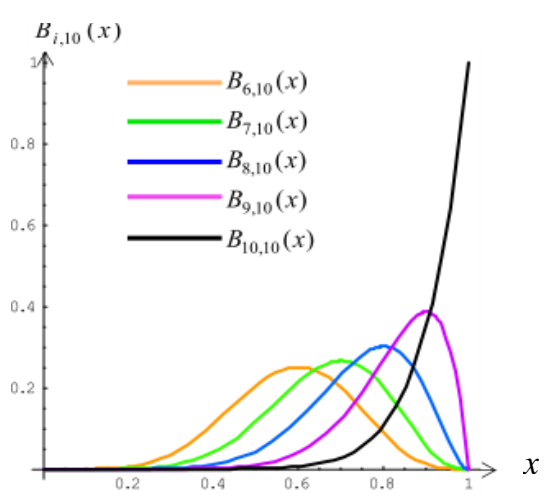

Fig. 1(b). Graph of last 5 Bernstein polynomials over $[0,1]$.

\section{Formulation of Integral Equation in Matrix Form}

Consider a general linear Fredholm integral equation (FIE) of second kind [1, 2] is given by

$$
a(x) \phi(x)+\lambda \int_{a}^{b} k(t, x) \phi(t) d t=f(x), a \leq x \leq b
$$

where $a(x)$ and $f(x)$ are given functions, $k(t, x)$ is the kernel, and $\phi(x)$ is the unknown function or exact solution of (2), which is to be determined.

Now we use the technique of Galerkin method [Lewis, 3] to find an approximate solution $\tilde{\phi}(x)$ of (2). For this, we assume that

$$
\tilde{\phi}(x)=\sum_{i=0}^{n} a_{i} B_{i, n}(x)
$$

where $B_{i, n}(x)$ are Bernstein polynomials (basis) of degree $i$ defined in eqn. (1), and $a_{i}$ are unknown parameters, to be determined. Substituting (3) into (2), we obtain

$$
a(x) \sum_{i=0}^{n} a_{i} B_{i, n}(x)+\lambda \int_{a}^{b}\left[k(t, x) \sum_{i=0}^{n} a_{i} B_{i, n}(t)\right] d t=f(x)
$$

or, 


$$
\sum_{i=0}^{n} a_{i}\left[a(x) B_{i, n}(x)+\lambda \int_{a}^{b} k(t, x) B_{i, n}(t) d t\right]=f(x)
$$

Then the Galerkin equations [Lewis, 3] are obtained by multiplying both sides of (3) by $B_{j, n}(x)$ and then integrating with respect to $x$ from $a$ to $b$, we have

$$
\sum_{i=0}^{n} a_{i}\left[\int_{a}^{b}\left[a(x) B_{i, n}(x)+\lambda \int_{a}^{b} k(t, x) B_{i, n}(t) d t\right] B_{j, n}(x) d x\right]=\int_{a}^{b} B_{j, n}(x) f(x) d x, j=0,1, \cdots, n
$$

Since in each equation, there are three integrals. The inner integrand of the left side is a function of $x$ and $t$, and is integrated with respect to $t$ from $a$ to $b$. As a result the outer integrand becomes a function of $x$ only and integration with respect to $x$ yields a constant. Thus for each $j(=0,1, \ldots, n)$ we have a linear equation with $n+1$ unknowns $a_{i}(i=0,1, \ldots, n)$. Finally (5a) represents the system of $n+1$ linear equations in $n+1$ unknowns, are given by

$$
\sum_{i=0}^{n} a_{i} C_{i, j}=F_{j}, \quad j=0,1,2, \cdots, n
$$

where

$$
\begin{aligned}
& C_{i, j}=\int_{a}^{b}\left[a(x) B_{i, n}(x)+\lambda \int_{a}^{b} k(t, x) B_{i, n}(t) d t\right] B_{j, n}(x) d x, \quad i, j=0,1,2, \ldots, n . \\
& F_{j}=\int_{a}^{b} B_{i, n}(x) f(x) d x, \quad j=0,1,2, \cdots, n
\end{aligned}
$$

Now the unknown parameters $a_{i}$ are determined by solving the system of equations (5), and substituting these values of parameters in (3), we get the approximate solution $\widetilde{\phi}(x)$ of the integral equation (2). The absolute relative error $E$ for this formulation is defined by

$$
E=\left|\frac{\varphi(x)-\tilde{\varphi}(x)}{\varphi(x)}\right| .
$$

\section{Numerical Examples}

In this section, we explain three integral equations which are available in the existing literatures $[1,2,6]$. For each example we find the approximate solutions using different number of Bernstein polynomials. 
Example 1: We consider the FIE of 2nd kind given by [6]

$$
\phi(x)-\int_{-1}^{1}\left(x t+x^{2} t^{2}\right) \phi(t) d t=1, \quad-1 \leq x \leq 1
$$

having the exact solution, $\phi(x)=1+\frac{10}{9} x^{2}$

Using the formulation described in the previous section, the equations (5) lead us, respectively,

$$
\begin{gathered}
\sum_{i=0}^{n} a_{i} \quad C_{i, j}=F_{j}, \quad j=0,1,2, \ldots, n, \\
C_{i, j}=\int_{-1}^{1} B_{i, n}(x) B_{j, n}(x) d x-\int_{-1}^{1}\left[\int_{-1}^{1}\left(x t+x^{2} t^{2}\right) B_{i, n}(t) d t\right] B_{j, n}(x) d x, \quad i, j=0,1, \ldots, n \\
F_{j}=\int_{-1}^{1} B_{j, n}(x) d x, \quad j=0,1,2, \ldots, n
\end{gathered}
$$

Solving the system (7) for $n=3$, the values of the parameters are:

$$
a_{0}=\frac{19}{9}, a_{1}=\frac{17}{27}, a_{2}=\frac{17}{27}, a_{3}=\frac{19}{9}
$$

Substituting into (3) and for $n \geq 3$, the approximate solution is,

$$
\tilde{\phi}(x)=1+\frac{10}{9} x^{2}
$$

which is the exact solution.

Example 2: Now we consider another FIE of 2nd kind given by [6]

$$
\phi(x)-\int_{-1}^{1}\left(x^{4}-t^{4}\right) \phi(t) d t=x, \quad-1 \leq x \leq 1
$$

having the exact solution $\phi(x)=x$

Proceeding as the example 1, the system of equations becomes as

$$
\sum_{i=0}^{n} a_{i} C_{i, j}=F_{j}, \quad j=0,1, \ldots, n
$$


where,

$$
C_{i, j}=\int_{-1}^{1} B_{i, n}(x) B_{j, n}(x) d x-\int_{-1}^{1}\left[\int_{-1}^{1}\left(x^{4}-t^{4}\right) B_{i, n}(t) d t\right] B_{j, n}(x) d x \quad i, j=0,1,2, \ldots, n,
$$

(9b)

$$
F_{j}=\int_{-1}^{1} x B_{j, n}(x) d x, \quad j=0,1, \ldots, n
$$

Now solving the system (9) for $n=3$, the values of the parameters, $a_{i}$ are:

$$
a_{0}=-1, a_{1}=-\frac{1}{3}, a_{2}=\frac{1}{3}, a_{3}=1
$$

and the approximate solution, for $n \geq 3$, is $\tilde{\phi}(x)=x$ which is the exact solution.

Example 3: Consider another FIE of 2nd kind given by [1, pp 213]

$$
\phi(x)-\int_{0}^{1}\left(t x^{2}+x t^{2}\right) \phi(t) d t=x, \quad 0 \leq x \leq 1
$$

having the exact solution $\phi(x)=\frac{180}{119} x+\frac{80}{119} x^{2}$

Proceeding as the previous examples, the system of equations becomes:

$$
\begin{aligned}
C_{i, j} & =\int_{0}^{1} B_{i, n}(x) B_{j, n}(x) d x-\int_{0}^{1}\left[\int_{0}^{1}\left(t x^{2}+x t^{2}\right) B_{i, n}(t) d t\right] B_{j, n}(x) d x, i, j=0,1, \ldots, n \\
F_{j} & =\int_{0}^{1} x B_{j, n}(x) d x, \quad j=0,1, \ldots, n
\end{aligned}
$$

For $n=3$, solving system (11), the values of the parameters $\left(a_{i}\right)$ are:

$$
a_{0}=0, a_{1}=\frac{260}{119}, a_{2}=\frac{80}{119}, a_{3}=0
$$

and the approximate solution is $\tilde{\phi}(x)=\frac{180}{119} x+\frac{80}{119} x^{2}$, which is the exact solution. 
Example 4: Consider another FIE of 2nd kind given by [2, pp 124]

$$
\phi(x)-\int_{0}^{1} 2 e^{x} e^{t} \phi(t) d t=e^{x}, \quad 0 \leq x \leq 1,
$$

having the exact solution $\phi(x)=\frac{e^{x}}{2-e^{2}}$.

Since the equations (5b) and (5c) are of the form

$$
\begin{aligned}
C_{i, j} & =\int_{0}^{1} B_{i, n}(x) B_{j, n}(x) d x-\int_{0}^{1}\left[\int_{0}^{1} 2 e^{x} e^{t} B_{i, n}(t) d t\right] B_{j, n}(x) d x \quad i, j=0,1,2, \ldots, n \\
F_{j} & =\int_{0}^{1} e^{x} B_{j, n}(x) d x, \quad j=0,1, \ldots, n
\end{aligned}
$$

For $n=3,4,5$, and 6, the approximate solutions are, respectively

$$
\begin{aligned}
& \tilde{\phi}(x)=-0.185387-0.188957 x-0.078167 x^{2}-0.051702 x^{3} \\
& \tilde{\phi}(x)=-0.185571-0.185273 x-0.0947442 x^{2}-0.0259153 x^{3}-0.0128933 x^{4} \\
& \tilde{\phi}(x)=-0.185561-0.18558 x-0.0926328 x^{2}-0.0315503 x^{3}-0.00655026 x-0.00253837 x^{5} \\
& \tilde{\phi}(x)=-0.185561-0.18556 x-0.0925932 x^{2}-0.0308581 x^{3}-0.00791665 x^{4} \\
& \quad-0.0012903 x^{5}-0.000427923 x^{6}
\end{aligned}
$$

Plots of absolute relative the errors $E$ between exact and approximate solutions are given in Fig. 2 for various values of $n$. One observes that the minimum order of accuracies are $10^{-4}, 10^{-5}, 10^{-7}$, and $10^{-5}$, respectively, with $4,5,6$ and 7 Bernstein polynomials. This confirms that if we increase the number of polynomials to more than 6 , the minimum accuracy is $10^{-7}$ (obtained using 6 polynomials), which guarantees the convergence. Now the approximate solutions, exact solutions, and the absolute relative error $E$, between exact and the approximate solutions at various points of the domain are shown in Table 1
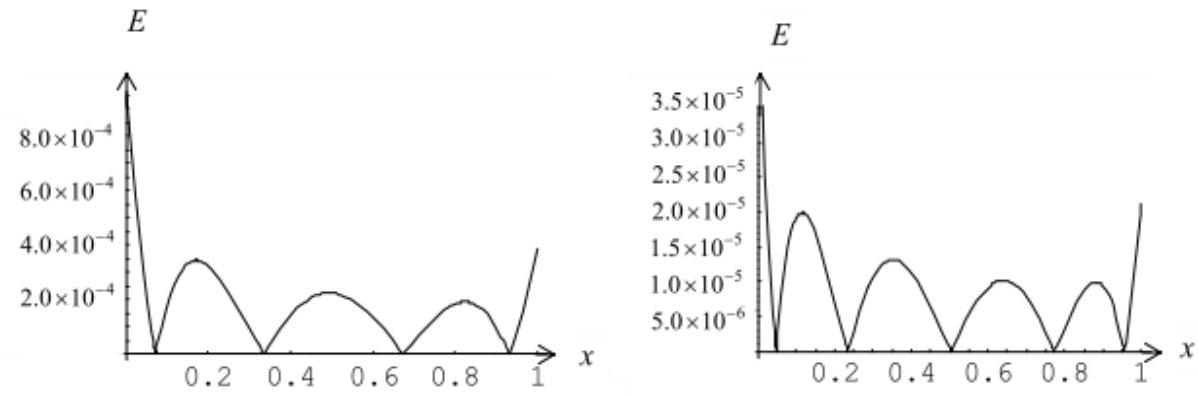
Fig. 2 (a). Using 4 polynomials .

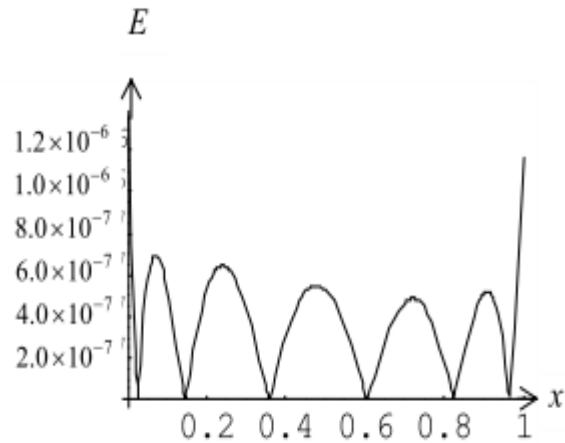

Fig. 2 (c). Using 6 polynomials .
Fig.2 (b). Using 5 polynomials.

\section{E}

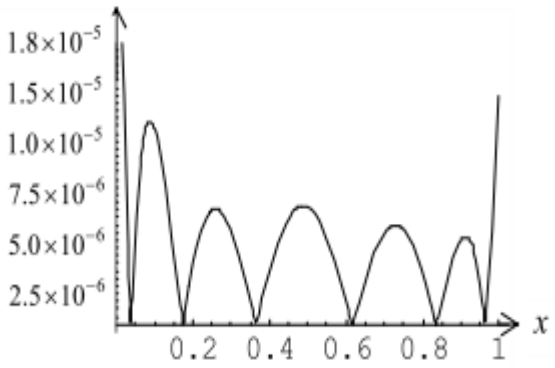

Fig.2 (d). Using 7 polynomials.

Table 1. Exact and approximate solutions and absolute relative errors of example 4.

\begin{tabular}{|c|c|c|c|c|c|}
\hline \multirow{2}{*}{$x$} & \multirow{2}{*}{$\begin{array}{c}\text { Exact } \\
\text { Solutions }\end{array}$} & $\begin{array}{l}\text { Approximate } \\
\text { Solutions }\end{array}$ & $\begin{array}{l}E \text {, Absolute } \\
\text { Relative Error }\end{array}$ & $\begin{array}{l}\text { Approximate } \\
\text { Solutions }\end{array}$ & $\begin{array}{l}\text { E, Absolute } \\
\text { Relative Error }\end{array}$ \\
\hline & & \multicolumn{2}{|c|}{ Polynomials used 4} & \multicolumn{2}{|c|}{ Polynomials used 5} \\
\hline 0.0 & -0.1855612526 & -0.1853868426 & $9.399053 \times 10^{-4}$ & -0.1855710276 & $5.267824 \times 10^{-5}$ \\
\hline 0.1 & -0.2050768999 & -0.2051159200 & $1.902705 \times 10^{-4}$ & -0.2050729953 & $1.903950 \times 10^{-5}$ \\
\hline 0.2 & -0.2266450257 & -0.2267185494 & $3.244002 \times 10^{-4}$ & -0.2266433896 & $7.218900 \times 10^{-6}$ \\
\hline 0.3 & -0.2504814912 & -0.2505049431 & $9.362730 \times 10^{-5}$ & -0.2504841183 & $1.048837 \times 10^{-5}$ \\
\hline 0.4 & -0.2768248595 & -0.2767853131 & $1.428573 \times 10^{-4}$ & -0.2768280333 & $1.146489 \times 10^{-5}$ \\
\hline 0.5 & -0.3059387842 & -0.3058698717 & $2.252492 \times 10^{-4}$ & -0.3059389305 & $4.784034 \times 10^{-7}$ \\
\hline 0.6 & -0.3381146470 & -0.3380688310 & $1.355042 \times 10^{-4}$ & -0.3381115499 & $9.159888 \times 10^{-6}$ \\
\hline 0.7 & -0.3736744748 & -0.3736924032 & $4.797873 \times 10^{-5}$ & -0.3736715753 & $7.759438 \times 10^{-6}$ \\
\hline 0.8 & -0.4129741624 & -0.4130508005 & $1.855761 \times 10^{-4}$ & -0.4129756348 & $3.565315 \times 10^{-6}$ \\
\hline 0.9 & -0.4564070342 & -0.4564542350 & $1.034182 \times 10^{-4}$ & -0.4564113003 & $9.347079 \times 10^{-6}$ \\
\hline \multirow[t]{2}{*}{1.0} & -0.5044077810 & -0.5042129189 & $3.863186 \times 10^{-4}$ & -0.5043970878 & $2.119953 \times 10^{-5}$ \\
\hline & & \multicolumn{2}{|c|}{ Polynomials used 6} & \multicolumn{2}{|c|}{ Polynomials used 7} \\
\hline 0.0 & -0.1855612526 & -0.1855612526 & $1.358183 \times 10^{-6}$ & -0.1855612694 & $4.157210 \times 10^{-5}$ \\
\hline 0.1 & -0.2050768999 & -0.2050768100 & $5.311130 \times 10^{-7}$ & -0.2050768958 & $1.272207 \times 10^{-5}$ \\
\hline 0.2 & -0.2266450257 & -0.2266450257 & $5.268384 \times 10^{-7}$ & -0.2266450312 & $3.763352 \times 10^{-6}$ \\
\hline 0.3 & -0.2504814912 & -0.2504814912 & $4.307390 \times 10^{-7}$ & -0.2504814909 & $6.065680 \times 10^{-6}$ \\
\hline 0.4 & -0.2768248595 & -0.2768248596 & $2.997736 \times 10^{-7}$ & -0.2768248544 & $3.529983 \times 10^{-6}$ \\
\hline 0.5 & -0.3059387842 & -0.3059387842 & $5.281912 \times 10^{-7}$ & -0.3059387842 & $7.651093 \times 10^{-6}$ \\
\hline 0.6 & -0.3381146470 & -0.3381146470 & $3.685661 \times 10^{-8}$ & -0.3381146522 & $1.444431 \times 10^{-6}$ \\
\hline 0.7 & -0.3736744748 & -0.3736744748 & $4.654666 \times 10^{-7}$ & -0.3736744750 & $5.945203 \times 10^{-6}$ \\
\hline 0.8 & -0.4129741624 & -0.4129741624 & $1.890301 \times 10^{-7}$ & -0.4129741564 & $3.339474 \times 10^{-6}$ \\
\hline 0.9 & -0.4564070342 & -0.4564070342 & $5.100054 \times 10^{-7}$ & -0.4564070387 & $5.480605 \times 10^{-6}$ \\
\hline 1.0 & -0.5044077810 & -0.5044077810 & $1.161812 \times 10^{-6}$ & -0.5044077618 & $1.495842 \times 10^{-5}$ \\
\hline
\end{tabular}

\section{Conclusion}


We have considered the integral equations to solve numerically. We have obtained the approximate solution of the unknown function by the well known Galerkin method using Bernstein polynomials as trial functions. We have verified the derived formulas with the appropriate numerical examples. In this context we may note that the numerical solutions coincide with the exact solutions even a few of the polynomials are used in the approximation.

\section{References}

1. A. J. Jerri, Introduction to Integral Equations with Applications (John Wiley \& Sons Inc. 1999).

2. S. Swarup, Integral Equations (Krishna Prakashan Media Pvt. Ltd, $15^{\text {th }}$ Edition, 2007).

3. P. E. Lewis and J. P. Ward, The Finite Element Method, Principles and Applications (AddisonWesley, 1991).

4. J. Reinkenhof, Int. J. Numer. Methods Engrg. 11, 1627 (1986). doi:10.1002/nme.1620111012

5. E. Kreyszig, Int. J. Numer. Methods Engrg. 14, 292 (1979). doi:10.1002/nme.1620140213

6. B. N. Mandal and S. Bhattacharya, Appl. Math.Comput. 190, 1707 (2007). doi:10.1016/j.amc.2007.02.058

7. M. I. Bhatti and P. Bracken, J. Comput. Appl. Math. 205, 272 (2007). doi:10.1016/j.cam.2006.05.002 